\title{
Distribución geográfica y mesológica de las especies piscícolas de la cuenca del Duero en el verano de 1981
}

\author{
Diego García de Jalón y José Vicente López Alvarez
}

Departamento de Zoología y Entomología, Escuela de Ingenieros de Monites, Ciudad Universitaria, Madrid-3

\section{SUMMARY \\ GEOGRAPHIC AND MESOLOGIC DISTRIBUTION OF FISHES IN THE DUERO RIVER BASIN (SPAIN) DURING SUMMER 1981}

Using electro-fishing seventeen species of fishes were found in the watershed of Duero river during a survey of 70 sampling stations carried out in summer 1981. Some physical and chemical parameters of the water were analyzed (alcalinity, hardness, conductivity, dissolved oxygen, nitrates, nitrites, phospates, $\mathrm{pH}$ and temperatures) and related to the presence of different species.

\section{INTRODUCCION}

La sequía estival, peculiaridad de nuestros climas mediterráneos, caracteriza los ecosistẹmas fluviales ibéricos, cuyo más claro exponente es el descenso del caudal con ello la contaminación de los ríos producida por vertidos urbanos, agricolas e industriales se hace máxima, al disminuir el factor dilución. En el presente trabajo se estudia el estado de las especies piscicolas de la cuenca del río Duero en las condiciones críticas del verano de 1981.

Resulta soprendente la escasez de trabajos que tratan de la distribución de peces en el interior de la península ibérica, especialmen- te si pensamos que la mayoría de las especies son objeto de pesca intensiva tanto deportiva como comercial. Como antecedentes relativos a la cuenca del Duero podemos citar los trabajos de VELEZ DE MEDRANO (1947), ANONIMUS (1952), LOZANO REY (1952), LOZANO CABO (1964), ALMACA (1976), ELVIRA et al. (en prensa) y DOADRIO \& ELVIRA (en prensa). Queremos también resaltar el olvido que han sufrido los estudios españoles y portugueses en obras tan importantes como el Catálogo Europeo de Peces de Agua Dulce (BANARESCU et al. 1971), la Limnofauna Europea (ILLIES, 1978) y las guías de MUUS \& DAHLSTROM (1970) y MAITLAND (1980). 


\section{METODOS}

Durante lo's meses de verano hemos realizado un muestreo de peces, así como análisis físico-químicos de las aguas, en 70 estaciones distribuidas por toda la cuenca del Duero cuya ubicación se ha elegido atendiendo a que estén repartidas homogéneamente por toda la zona de estudio y evitando los tramos intensamente contaminados.

El muestreo piscícola se ha realizado principalmente mediante pesca eléctrica (corriente continua de $1.000 \mathrm{~W}$ ) y accesoriamente con trasmallos. Los análisis de aguas se realizaron "in situ" con un laboratorio portátil Hach, calculando los valores de los siguientes parámetros: alcalinidad, dureza, conductividad, oxigeno disuelto, nitratos; nitritos, fosfatos y $\mathrm{pH}$. Las temperaturas de las aguas se tomaron con termómetros convencionales.

\section{CATALOGO DE ESPECIES}

En el presente catálogo indicamos, para cada especie, las localidades de la cuenca del Duero donde hemos encontrado ejemplares durante el verano de 1981. Entre paréntesis se añaden las abreviaturas de las provincias a las que pertenece cada localidad. AV: Avila, BU: Burgos, LE: León, PAi Palencia, SA: Salamanca, SG: Segovia, SO: Soria, VA: Valladolid y ZA: Zamora.

\section{FAMILIA ANGUILLIDAE} (SA)

Anguilla anguilla (Linnaeus, 1758)

Río Agueda: Castillejo de Martín Viejo

\section{FAMILIA SALMONIDÁE}

Salmo trutta fario (Linnaeus, 1758)

La trucha se encuentra en las zonas más altas de la cuenca, a una altitud media superior a los $1.000 \mathrm{~m}$. sobre el nivel del mar (fig. 1).
Río Duero: Duruelo de la Sierra (SO); Garray (SO); Tardajos de Duero (SO). Rio Ucero: Ucero (SO). Río Pisuerga: Polentinos (PA); Alar del Rey (PA); Río Arlanzón: Pineda de la Sierrra (BU); San Millán de Juarros (BU). Rio Arlanza: Quintanar de la Sierra (BU). Río Carrión: Triollo (PA); Pino del Rio (PA); Villoldo (PA). Río Esla: Riano (LE); Gradefes (LE); Mansilla de las Mulas (LE); Santa Colomba de las Carabias (ZA); Bretó (ZA). Río Cea: Almanza (LE). Río Porma: Puebla de Lillo (LE); Vegas del Condado (LE). Rio Bernesga: La Vid (LE). Río Orbigo: Rabanal de Luna (LE); Carrizo de la Ribera (LE); Requejo de la Vega.(LE). Rio Tera: Trefacio (ZA); Mozar (ZA). Rio Riaza : Riofró (SG); Berlangas de Roa (BU) Río Duratón: Duruelo (SG). Río Eresma: Valsain (SG). Rio Tormes; Angostura (AV); Ejeme (SA). Río Agueda: El Payo (SA).

\section{FAMILIA ESOCIDAE}

Esox lucius (Linnaeus, 1758)

Río Esla: Bretó (ZA).

\section{FAMILIA CYPRINIDAE}

\section{Cyprinus carpio (Linnaeus, 1758)}

La carpa se ha encontrado entre los $300 y$ $750 \mathrm{~m}$. de altitud, en zona de Potamon característico (fig. 1).

Río Duero: Villamarciel (VA); Castronuño (VA); Peleagonzalo (ZA); Carrascal (ZA). Río Carrión: Villamuriel de Cerrato (PA). Rio Tormes: Ledesma (SA)

Barbus barbus bocagei (Steindachner, 1865)

El intervalo de altitud en que se ha encontrado el barbo es muy amplio, desde los 190 m. hasta los $1.180 \mathrm{~m}$. (fig. 1).

Río Duero: Garray (SO); Langa de Duero (SO); Peleagonzalo (ZA); Río Ucero: Burgo de Osma (SO). Río Pisuerga: Cordovilla la Real (PA); Soto de Cerrato (PA); Cabezón (VA). Río Arlanzón: San Millán de Juarros (BU). Río Arlanza: Torrepadre (BU); Quintana del Puente (PA). Río Esgueva: Cabanes de Esgueva (BU). Río Carrión: Villoldo (PA); Villamuriel de Cerrato (PA). 
Rio Valderaduey: Benegiles (ZA). Rio Esla: Gradefes (LE); Mansilla de las Mulas (LE); Ardón (LE); Santa Colomba de las Carabias (ZA); Bretó (ZA). Río Cea: Almanza (LE). Río Orbigo: Requejo de la Vega (LE); Santa Cristina de la Polvorosa (ZA). Río Tera: Trefacio (ZA). Rio Eresma: Hornillos (VA). Río Adaja: Valdestillas (VA). Rio Tormes: Angostura (AV); Ejeme (SA). Rio Huebra: Tamames (SA); Cerralbo (SA). Río Agueda: El Payo (SA); Castillejo de Martín Viejo (SA); La Fregeneda (SA).

Carassius auratus (Linnaeus, 1758)

El carpín es otra especie caracteristica del Potamon bajo del Duero. Se ha encontrado a una altitud media de $530 \mathrm{~m}$. (fig. 1).

Río Duero: Peleagonzalo (ZA); Carrascal (ZA); La Fregeneda (SA). Rio Pisuerga: Cabezón (VA). Rio Valderaduey: Benegiles (ZA). Río Tormes: Ledesma (SA). Río Huebra: Cerralbo (SA). Río Agueda: La Fregeneda (SA). 1866)

Chondrostoma polylepis (Steindachner,

La boga la hemos encontrado distribuida por toda la cuenca, desde los $190 \mathrm{~m}$. hasta los $1.200 \mathrm{~m}$. de altitud (fig. 1).

Río Duero: Hinojosa de la Sierra (SO); Garray (SO); Tardajos de Duero (SO); Castronuño (VA); Peleagonzalo (ZA); La Fregeneda (SA). Río Ucero: Ucero (SO). Río Pisuerga: Alar del Rey (PA); Cordovilla la Real (PA); Soto de Cerrato (PA); Cabezón (VA). Río Esgueva: Cabanes de Esgueva (BU). Río Arlanza: Torrepadre (BU); Quintana del Puente (PA). Río Arlanzón: San Millán de Juarros (BU). Río Carrión: Villoldo (PA). Río Valderaduey: Benegiles (ZA). Río Esla: Mansilla de las Mulas (LE); Santa Colomba de las Carabias (ZA). Río Cea: Almanza (LE). Río Orbigo: Rabanal de Luna (LE); Requejo de la Vega (LE); Santa Cristina de la Polvorosa (ZA). Rio Tera: Trefacio (ZA). Río Eresma:"Hornillos (VA). Rio Adaja: Blascosancho (AV). Río Tormes: Angostura (AV); Ejeme (SA). Rio Huebra: Cerralbo (SA). Río Agueda: El Payo (SA); Castillejo de Martín Viejo (SA); La Fregeneda (SA).
Gobio gobio (Linnaeus, 1758)

Su distribución es análoga a la de la boga (fig. 1).

Río Duero: Garray (SO); Tardajos de Duero (SO); Langa de Duero (SO); Carrascal (ZA). Rio Ucero: Ucero (SO). Río Pisuerga: Polentinos (PA); Alar del Rey (PA); Cordovilla la Real (PA); Soto de Cerrato (PA); Cabezón (VA). Río Arlanzón: San Millán de Juarros (BU). Río Arlanza: Quintanar de la Sierra (BU); Torrepadre (BU); Quintana del Puente (PA). Río Esgueva: Cabanes de Esgueva (BU). Río Carrión: Pino del Rio (PA); Villoldo (PA). Río Esla: Gradefes (LE); Mansilla de las Mulas (LE); Ardón (LE); Santa Colomba de las Carabias (ZA); Bretó (ZA). Río Cea: Almanza (LE). Río Orbigo: Rabanal de Luna (LE); Carrizo de la Ribera (LE); Requejo de la Vega (LE); Santa Cristina de la Polvorosa (ZA). Rio Tera: Trefacio (ZA); Mozar (ZA). Río Riaza: Berlangas de Roa (BU); Río Duratón: Duruelo (SG); Laguna de Contreras (SG). Rio Cega: La Pedraja del Portillo (VA). Rio Eresma: Bernardos (SG); Hornillos (VA). Río Adaja: Blascosancho (AV); Valdestillas (VA). Río Trabancos: Sieteiglesias (VA). Rí Tormes: Ejeme (SA). Rio Agueda: La Fregeneda (SA).

Leuciscus cephalus (Linnaeus, 1758)

La distribución del cacho es semejante a la de la boga y el gobio (fig. 1).

Río Duero: Hinojosa de la Sierra (SO); Garray (SO); Langa de Duero (SO); Castronuño (VA); Peleagonzalo (ZA); Carrascal (ZA). Río Pisuerga: Polentinos (PA); Cordovilla la Real (PA); Cabezón (VA). Rio Esgueva: Cabanes de Esgueva (BU). Río Arlanza: Torrepadre (BU); Quintana del Puente (PA). Río Arlanzón: San Millán de Juarros (BU). Rio Carrión: Villoldo (PA). Rio Valderaduey: Benegiles (ZA). Rio Esla: Gradefes (LE); Mansilla de las Mulas (LE); Santa Colomba de las Carabias (ZA). Río Cea: Almanza (LE). Río Porma: Puebla de Lillo (LE). Río Orbigo: Requejo de la Vega (LE); Santa Cristina de la Polvorosa (ZA). Rio Tera: Trefacio (ZA). Rio Duratón: Duruelo (SG). Río Eresma: Bernardos (SG); Hornillos (VA). Río Tormes: Angostura 
(AV); Ejeme (SA). Río Huebra: Cerralbo (SA). Río Agueda: El Payo (SA); Castillejo de Martín Viejo (SA); La Fregeneda (SA).

Phoxinus phoxinus (Linnaeus, 1758)

El piscardo se ha encontrado en los tramos de la vertiente NE de la cuenca.

Río Ucero: Ucero (SO). Río Pisuerga: Polentinos (PA). Río Arlanza: Quintanar de la Sierra (SO). Río Arlanzón: San Millán de Juarros (BU).

Rutilus albumoides (Steindachner, 1866)

El calandino sólo se ha encontrado en el río Agueda.

Rio Agueda: El Payo (SA); Castillejo de Martín Viejo (SA); La Fregeneda (SA).

Rutilus lemmingii (Steindachner, 1866)

La pardilla se ha encontrado exclusivamente en el río Huebra, cuyas aguas tienen un carácter marcadamente temporal. (SA).

Río Huebra: Tamames (SA); Cerralbo

Rutilus rubilio arcasii (Steindachner, 1892)

La bermejuela se encuentra repartida por toda la cuenca salvo en el cuadrante SW, donde viven el calandino y la pardilla, en altitudes comprendidas entre 640 y $1.300 \mathrm{~m}$. de altitud (fig. 1).

Río Duero: Duruelo de la Sierra (SO); Hinojosa de la Sierra (SO); Garray (SO); Tardajos (SO); Castronuño (VA); Peleagonzalo (ZA). Rio Ucero: Burgo de Osma (SO). Río Pisuerga: Polentinos (PA); Alar del Rey (PA); Soto de Cerrato (PA); Cabezón (VA). Rio Arlanza: Quintanar de la Sierra (BU); Torrepadre (BU); Quintana del Puente (PA). Río Arlanzón: San Millán de Juarros (BU). Río Carrión: Triollo (PA); Pino del Río (PA); Villoldo (PA); Villamuriel de Cerrato (PA). Río Valderaduey: Benegiles (ZA). Río Esir: Riaño (LE); Gradefes (LE); Mansilla de las Mulas (LE); Ardón (LE); Santa Colomba de las Carabias (ZA). Río Cea: Almanza (LE). Río Porma: Puebla de Lillo (LE). Río Orbigo: Rabanal de Luna (LE); Carrizo de la
Ribera (LE); Requejo de la Vega (LE). Río Duratón: Duruelo (SG); Laguna de Contreras (SG). Río Cega: Rebollo (SG); La Pedraja del Portillo (VA). Río Eresma: Bernardos (SG); Homillos' (VA). Río Adaja: Pradosegar (AV); Blascosancho (AV); Valdestillas (VA). Río Trabancos: Salvadiós (AV); Sieteiglesial (VA). Río Tormes: Ejeme (SA).

Tinca tinca (Linnaeus, 1758)

Rio Orbigo: Requejo de la Vega (LE). Río Huebra: Tamames (SA).

\section{FAMILIA COBITIDAE}

Cobitis calderoni (Bacescu, 1961)

La colmilleja se ha encontrado en los tramos altos y medio altos (700 a $1.200 \mathrm{~m}$. de altitud) estando ausente en el cuadrante SW de la cuenca.

Río Duero: Garray (SO). Río Ucero: Burgo de Osma (SO). Rio Pisuerga: Cabezón (VA). Río Arlanzón: San Millán de Juarros (BU). Río Arlanza: Quintanar de la Sierra (BU); Torrepadre (BU). Rio Carrión: Pino del Río (PA); Villoldo (PA). Río Esla: Riaño (LE); Gradefes (LE); Mansilla de las Mulas (LE); Ardón (LE). Rio Porma: Vegas del Condado (LE). Rio Bernesga: La Vid (LE). Río Orbigo: Rabanal de Luna (LE); Carrizo de la Ribera (LE); Requejo de la Vega (LE); Santa Cristina de la Polvorosa (ZA). Río Tera: Mozar (ZA). Río Duratón: Duruelo (SG); Laguna de Contreras (SG). Rio Cega: Rebollo (SG).

\section{FAMILIA POECILIIDAE}

Gambusia affinis (Baird \& Girard, 1853)

La gambusia se ha capturado en las localidades donde el $\mathrm{pH}$ de las aguas tenía los valores máximos de la cuenca $(8,5$ a 10,5).

Río Duero: Carrascal (ZA). Río Tormes: Ledesma (SA). Río Huebra: Cerralbo (SA). 


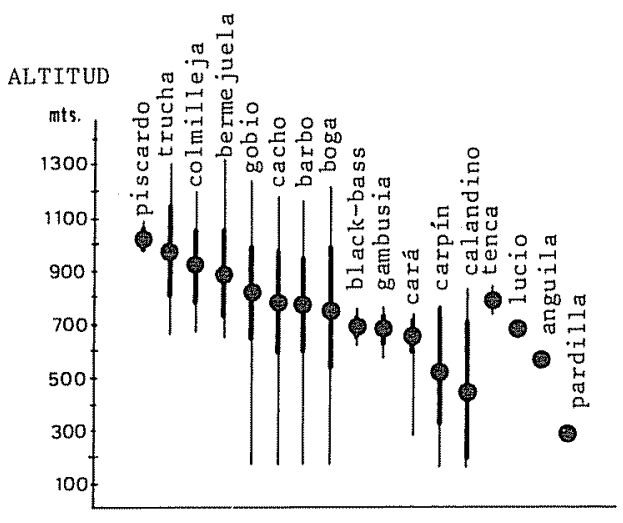

Fig. 1. Diagrama de los valores medios, máximos y mínimos e intervalo de altitud, para cada especie.

\section{FAMILIA CENTRARCHIDAE}

Micropterus salmoides (Lacepede, 1802)

Río Esla: Bretó (ZA). Río Orbigo: Santa Cristina de la Polvorosa (ZA). Rio Tera: Mozar (ZA).

\section{CARACTERISTICAS MESOLOGICAS}

Una vez expuesta la distribución de las especies piscícolas en el verano de 1981, en la cuenca del Duero, vamos a estudiar los in tervalos en que oscilan (en esas condiciones estivales) los principales parámetros físicoquímicos de las aguas, en las localidades consideradas.

Las circunstancias de la sequía produjeron un marcado descenso de los caudales e incluso su desaparición en largos tramos especialmente en los ríos Esgueva, Valderaduey, Cea, Cega, Duratón, Eresma, Adaja, Trabancos y Huebra. Es necesario señalar la modificación que suponen sobre estas condiciones naturales los numerosos embalses que se han construido en la cuenca del Duero, en especial los destinados a riegos, que en la época estival abren sus compuertas originando caudales considerables en los tramos de aguas abajo, que pueden igualar e incluso superar los caudales de invierno $y$ primavera. Este fenómeno sucede principalmente en los ríos Duero, Pisuerga, Carrión, Porma, Orbigo, Tera, Riaza, Duratón y Tormes. Por otra parte estas aguas que salen en verano de los grandes embalses, donde han sufrido una eutrofización a veces considerable, reciben las filtraciones de las aguas prócedentes de riegos de cultivos, cargadas de nutrientes, y de los vertidos industriales y urbanos. Por ello se produce un exuberante crecimiento de macrófitos, algas bentónicas y fitoplancton, cuya fotosintesis y respiración provocan unas fuertes oscilaciones diarias de diversos parámetros físico-químicos, en especial el $\mathrm{pH}$ y el oxígeno disuelto (HAWKES, 1973). En nuestro caso las oscilaciones de $\mathrm{pH}$ son especialmente intensas en el río Tormes, aguas abajo de Salamanca-ciudad. Las fuertes variaciones diarias de oxígeno disuelto han sido detectadas en los tramos medio y bajo de los ríos Duero y Pisuerga.

Los valores medios de la temperatura, encontrados para las distintas especies, (fig. 2) varían entre $16^{\circ} \mathrm{C}$ para la trucha y $23^{\circ} \mathrm{C}$ para la carpa. Más del $60 \%$ de las estaciones donde se han encontrado la trucha y la colmilleja están por debajo de $18^{\circ} \mathrm{C}$, aunque se encontraron a temperaturas de $25^{\circ} \mathrm{C}$ en el Tormes, que es el valor límite para la supervivencia de la trucha (ALABASTER \& LLOYD, 1980). En el río Tormes se han capturado carpas y carpines en aguas con temperaturas de $27^{\circ} \mathrm{C}$.

TEMPERATURA

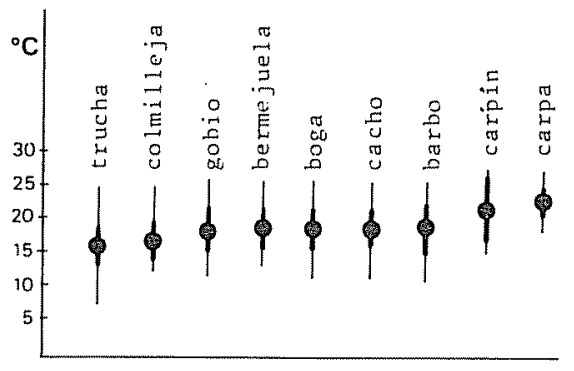

Fig. 2. Diagrama de los valores medios, máximos e intervalo de la temperatura, para cada especie. 
CONDUCTIVLDAD

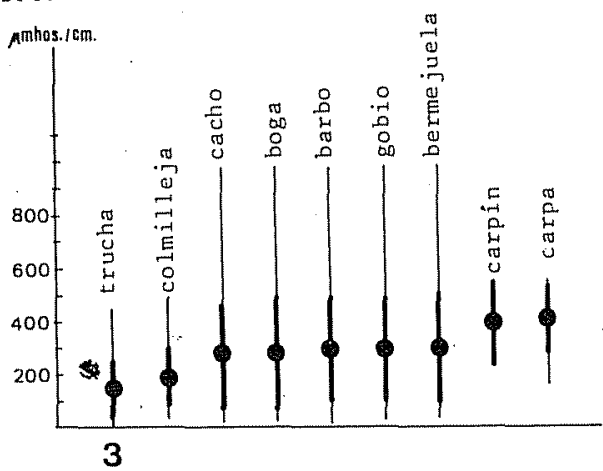

Fig. 3. Diagrama de los valores medios, máximos y mínimos e intervalo de la conductividad, para cada especie.

En el Duero los valores medios de la conductividad de las aguas donde se encontraban cada una de las especies (fig. 3) oscilan entre $150 \mu \mathrm{mhos} / \mathrm{cm}$. para la trucha y 400 $\mu \mathrm{mhos} / \mathrm{cm}$. para la carpa, límites aceptables para todos los peces. (BREMOND \& VUICHAR, 1973). Las poblaciones de trucha $y$ colmilleja no se encuentran con valores superiores a $500 \mu \mathrm{mhos} / \mathrm{cm}$. El resto de las especies tiene un intervalo muy amplio, con valores de conductividades de más de 1.000 $\mu \mathrm{mhos} / \mathrm{cm}$. en los ríos Arlanza y Adaja, donde viven la boga, cacho, barbo, gobio y bermejuela. La carpa y el carpín viven en aguas con unos intervălos más cortos de variación de la conductividad (entre $150 \mathrm{y}$ $550 \mu \mathrm{mhos} / \mathrm{cm}$ ); ello puede deberse a que sólo se han realizado capturas de estas especies en seis y ocho localidades, respectivamente.

Los valores de $\mathrm{pH}$ medidos en la cuenca del Duero son fundamentalmente básicos, comprendidos entre 6,5 y 11 (fig. 4). Los intervalos de este parámetro para cada especie son muy semejantes entre sí, con un valor medio próximo a 8 . La colmilleja presenta un intervalo más reducido que las restantes especies. Los tramos bajos de los ríos de la cuenca del Duero están en general eutrofizados, creciendo en ellos de forma abundante una vegetación acuática que produce durante el dia un aumento del

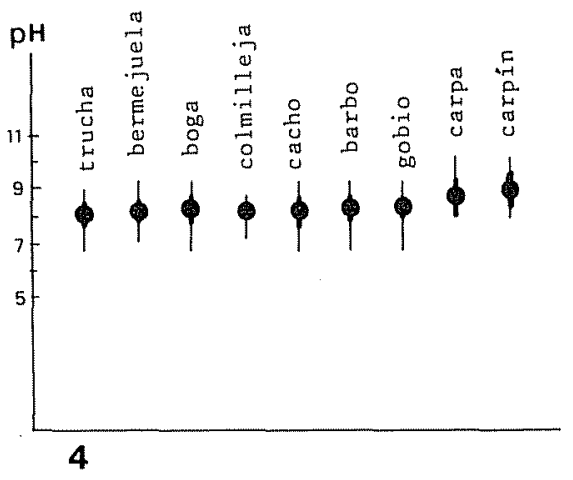

Fig. 4. Diagrama de los valores medios, máximos y mínimos e intervalo del $\mathrm{pH}$, para cada especie.

$\mathrm{pH}$ de las aguas. Los valores máximos de este factor se han registrado en los tramos bajos de los ríos de aguas blandas (Tormes, Huebra y Agueda) al estar poco tamponadas. Estos máximos de pH (entre 9,5 y 10,5) se aproximan a los valores letales para cualquier especie piscícola (ALABASTER \& LLOYD, 1980). Las especies más características de estas aguas básicas son la carpa, el carpín, la tenca, la gambusia y la pardilla.

El oxígeno disuelto en las aguas es un parámetro muy utilizado en los estudios de zonación piscicola y de contaminación. Conviene resaltar, no obstante, ciertas cuestiones que en general no se tienen en cuenta al considerar este parámetro y que aquí significamos (fig. 5).

La colmilleja y la trucha son las especies más exigentes en oxígeno disuelto ya que los valores de las estaciones en que aparecen se encuentran por encima de $7 \mathrm{mg} / 1$. Ello no implica que vivan en las aguas más oxigenadas; el valor máximo de oxígeno disuelto en las mismas $(13 \mathrm{mg} / 1)$ no corresponde al máximo de la cuenca $(17 \mathrm{mg} / 1)$. Tampoco el valor medio de este factor en las aguas donde aparecen estas especies es superior al de las estaciones en que aparecen las restantes especies.

Por el contrario, las especies más tolerantes a condiciones anóxicas (la carpa y el carpín) son las que se encuentran en las es- 


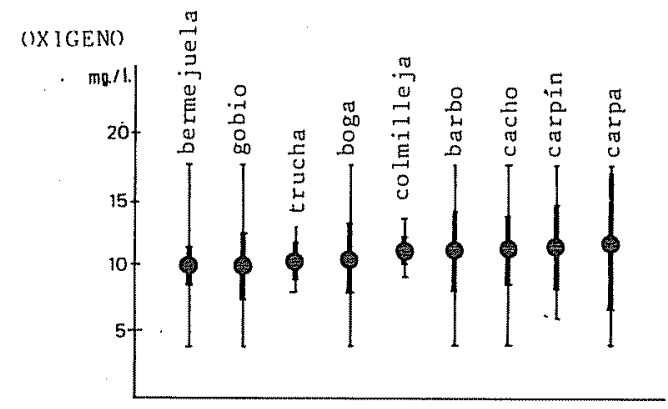

5
ALCALINIDAD

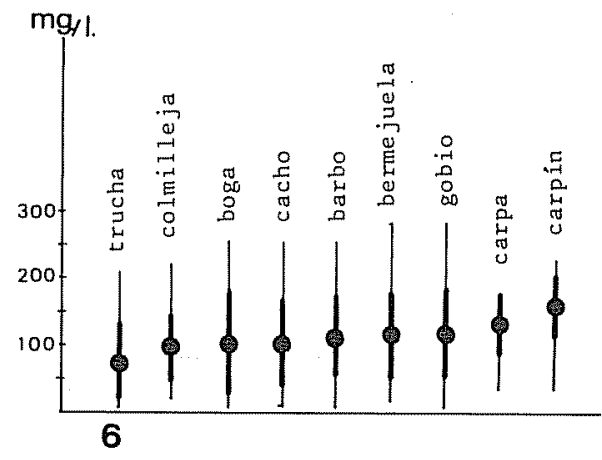

Fig. 6. Diagrama de los valores medios, máximos y mínimos e intervalo de la alalinidad, para cada especie.

predominar el efecto de esta última sobre el de la primera y, como consecuencia, existir condiciones de sobresaturación, con un máximo marcado al final del día $y$ un mínimo al amanecer.

Los valores medios de alcalinidad (fíg. 6) varian entre 70 y $160 \mathrm{mg} / 1 \mathrm{CO}_{3} \mathrm{Ca}$, correspondientes a una alcalinidad poco acusada o media (NISBET \& VERNEAUX, 1970). La trucha se mueve en aguas muy poco alcalinas, menos de $10 \mathrm{mg} / 1$ en el río Riaza, y también en aguas de $210 \mathrm{mg} / 1$ en el mismo río. La carpa vive en aguas con valores de alcalinidad

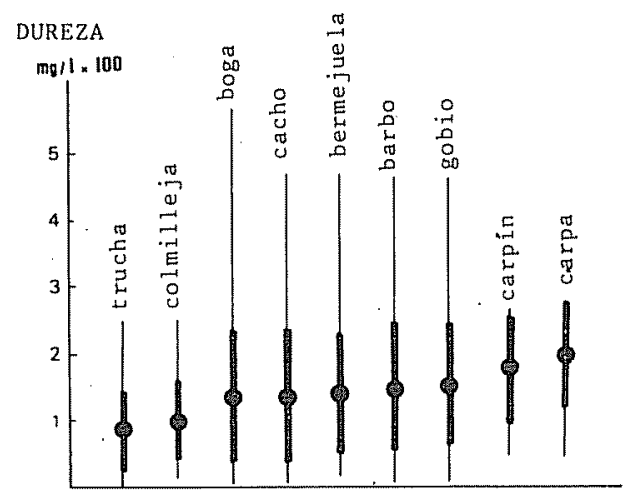

Fig. 7. Diagrama de los valores medios, máximos y mínimos e intervalo de la dureza, para cada especie.

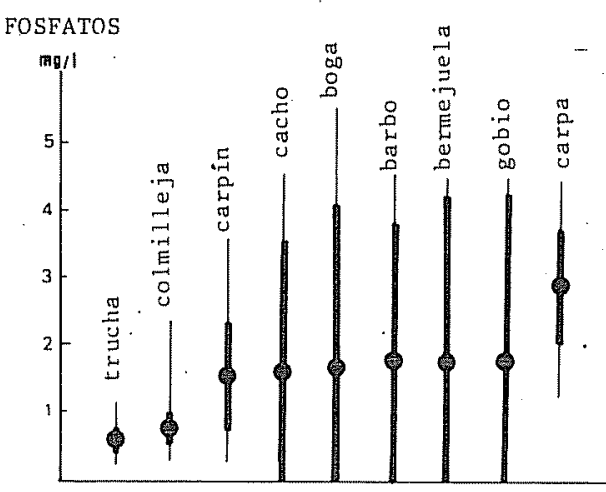

Fig. 8. Diagrama de los valores medios, máximos y mínimos e intervalo de la concentración de fosfatos en las aguas, para cada especie. 


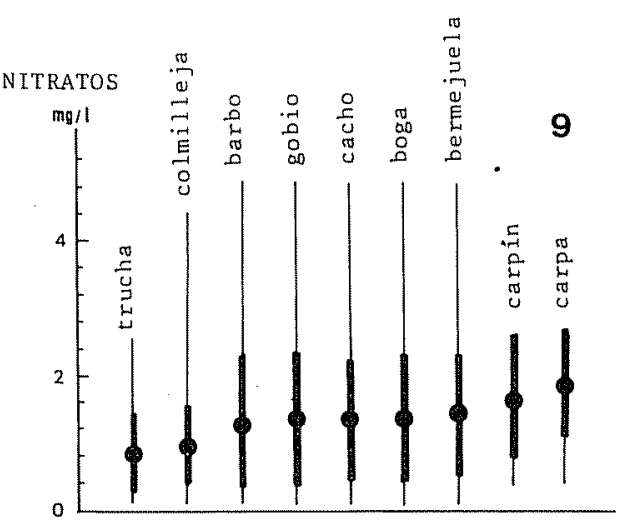

Fig. 9. Diagrama de los valores medios, máximos y mínimos e intervalo de las concentraciones de nitratos en las aguas, para cada especie.

de $40 \mathrm{mg} / 1$ en el Tormes y $180 \mathrm{mg} / 1$ en el Duero. El máximo absoluto se encuentra en aguas del Trabancos y Adaja $(275 \mathrm{mg} / 1$ ) donde viven la bermejuela y el gobio. La dureza de las aguas presenta, para cada especie, valores medios que oscilan entre 85 y $200 \mathrm{mg} / 1 \mathrm{CO}_{3} \mathrm{Ca}$ con variaciones iguales a las de la alcalinidad como era de esperar.

Todos los valores encontrados de fosfatos están por encima de $0,1 \mathrm{mg} / 1$. Si atendemos al valor límite indicador de aguas eutrofizadas, tanto al establecido por PRAT et al. (1981) de $0,1 \mathrm{mg} / 1$ ó bien al de NISBET \& VERNEAUX (1970) de 0,15 mg/1, podemos afirmar que prácticamente todas las aguas analizadas en la cuenca están eutrofizadas e incluso fuertemente eutrofizadas. La trucha y la colmilleja se presentan en aguas con intervalos menores de fosfatos, (entre $0,1 \mathrm{y}$ $1,2 \mathrm{mg} / 1$ para la trucha). El resto de las especies presenta una amplia variación de espectro en la cuenca siendo la carpa la que se presenta en aguas con un valor medio máximo de $3 \mathrm{mg} / 1$ típico de aguas contaminadas. (fig. 8).

La media de los valores de nitratos en las aguas (fig. 9) varía entre $0,8 \mathrm{mg} / 1$ para la trucha y $2 \mathrm{mg} / 1$ para la carpa. Los valores medios de nitritos en las aguas, (fig. 10) para

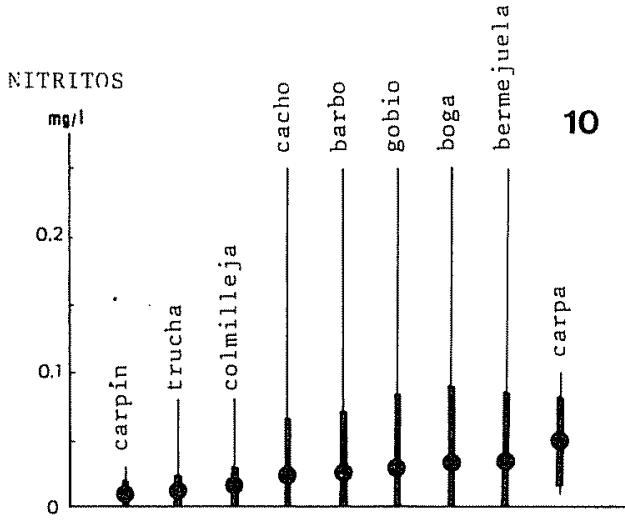

Fig. 10. Diagrama de los valores medios, máximos y mínimos e intervalo de la concentración de nitritos en las aguas, para cada especie.

cada especie, están comprendidos entre $0,013 \mathrm{mg} / 1$ para la trucha y $0,04 \mathrm{mg} / 1$ para la carpa. Los valores máximos $(0,1$ a 0,25 $\mathrm{mg} / 1)$ del intervalo de fluctuación de algunas especies (cacho; gobio, boga, bermeja y carpa) son considerados como de dudoso efecto para la fauna piscicola (TUFFERY, 1979). Hay que tener en cuenta que algunos de los valores bajos de nitritos en aguas eutróficas, caso de las aguas en que vive el carpin, se pueden explicar considerarndo que estos compuestos nitrogenados han pasado a formas amoniacales que no hemos estudiado.

\section{CONCLUSIONES}

En el muestreo estival realizado en la Cuenca del Duero hemos encontrado un total de 17 especies, de las cuales la trucha, el gobio, la bermejuela, la boga, el barbo y el cacho están distribuidas geográficamente por toda la Cuenca. La pardilla, el calandino y la anguila se encuentran restringidas al cuadrante SW de la Cuenca, las dos primeras por causas naturales son especies (vicarias de la bermejuela) y la última debido a la existencia de numerosas presas en el tramo bajo del 
Duero que impide su entrada más al interior. La colmilleja se ha encontrado en el área disjunta de las especies anteriores, $\mathrm{N}$ y SE de la cuenca. El piscardo se restringe al $\mathrm{NE}$, aunque se considera en expansión (LOZANO REY, 1952).

Respecto a su distribución altitudinal en época estival, las especies piscícolas se pueden agrupar en un conjunto que habita los tramos predominantemente altos (con más de $800 \mathrm{~m}$. de altitud), formado por el piscardo, la trucha, la colmilleja y la bermejuela; y en otro que se encuentra en los tramos bajos, constituido por la carpa, la gambusia, el "blackbass" y el carpín.
Desde un punto de vista mesológico podemos separar, por un lado, la trucha y la colmilleja (representantes del Rhithron) y, por otro, la carpa y el carpin (representantes del Potamon), que presentan intervalos de variación de los parámetros analizados muy pequeños, aunque con valores medios distintos, de las restantes especies de la cuenca, mucho más eurioicas que aquéllas.

\section{AGRADECIMIENTOS}

Agradecemos a I. Doadrio, B. Elvira, A. Palomares y J. Pajares su colaboración y ayuda prestada en los muestreos piscícolas.

BIBLIOGRAFIA

ALABASTER, J.S. \& LLOYD, R. 1980. Water Oua lity Criteria for Freshwater Fish. Butterwaths, London.

ALMACA, C: 1976. Zoogeografía e especiaçao dos Ciprinideos da Peninsula Iberica. Soc. Port. Cienc. Nat. Vol. 4: 1-28.

ANONIMUS, 1952. Las colecciones de peces de la Sección de Biologia de Aguas Continentales. IFIE, Madrid.

BANARESCU, P.; BLANC, C.; CAUDET, J.L. \& HUREAU, J.C. 1971. European Inland Water Fish. A multilingual Catalogue. Fishing News (Books) Ltd. London.

BREMOND, R. \& VUICHARD, R. 1973. Parame ters de la qualité des eaux. S.P.E.P.E. Doc Franc., París.

DOADRIO, I. \& EIVIRA, B. En prensa. Distribución geográfica de Rutilus arcasii (Steindachner, 1866) y Rutilus lemmingii (Steindachner 1866) (Pisces, Cyprinidae) en la península ibérica. Bol. Soc. Esp. Ecol. Biogeogr.

ELVIRA, B.; DOADRIO, I.; PALACIO, F.;VIGAL, C.R. \& LOBON, J. En prensa. Distribución geográfica de Chondrostoma polylepis Steindachner 1865 y Chondrostoma toxostoma (Vallot, 1837) (Pisces, Cyprinidae) en la península ibérica. Bol. Soc. Esp. Ecol. Biogeogr:

HAWKES, H.A. 1973. Eutrophication of rivers. Effects, causes and control. In: Treatment of Industrial Effluents. (A.G. CALLELY, C.F. FORSTER \& D.A. STAFFORD eds.): págs. 159-189. Hodder and Stoughton. London.

HAWKES, H.A. \& DAVIES, L.J. 1971. Some effects of organic enrichment on benthic inver- tebrate communities in stream riffles. In: The Scientific Management of Animal and Plant Communities for Conservation (E. DUFFEY and A.S. WATT eds.): págs. 271-293. Blackwell. Oxford.

ILLIES, J. 1978. Limnofauna Europaea. Fisher. Verlag, Stuttgart.

L.OZANO CABO, F. 1964. Los peces de las agtuas continentales españolas. S.N.P.F.C. Madrid.

LOZANO REY, L. 1952. Los peces fluviales de España. Ministerio de Agricultura. Madrid.

MAITLAND, P.S. 1980. Guía de los peces de agua dulce de Europa. Omega. Barcelona.

MUUS, B.J. \& DAHLSTROM, P. 1970. Los peces de agua dulce de España y de Europa. Omega. Barcelona.

NISBET, M. \& VERNEAUX, J. 1970. Composantes chimiques des eaux courantes. Discussion et proposition de classes en tant que bases d'interpretation des analy ses chimiques. Annls. Limnol. $6(2): 161-190$.

PRAT, N.; BAUTISTA, M.I.; GONZALEZ, G. \& PUIG, M.A. 1980. Eutrofització dels rius d'Andorra. Butll. Inst. Cat. Hist. Nat. 45: 107-114.

TUFFERY, G. 1979. Incidencias ecológicas de la polución de las aguas corrientes. Reveladores biológicos de la polución. In: La contaninación de las aguas continentales (P. PESSON, ed.): 215-253. Mundi Prensa. Madrid.

VELAZ DE MEDRANO, L. 1947. Dos notas sobre Ictiología Fluvial española. Localidades de B. barbus bocagei Steind. y $B$. comiza Steind. IFIE $\mathrm{N}^{\mathrm{O}} 36$. Madrid. 